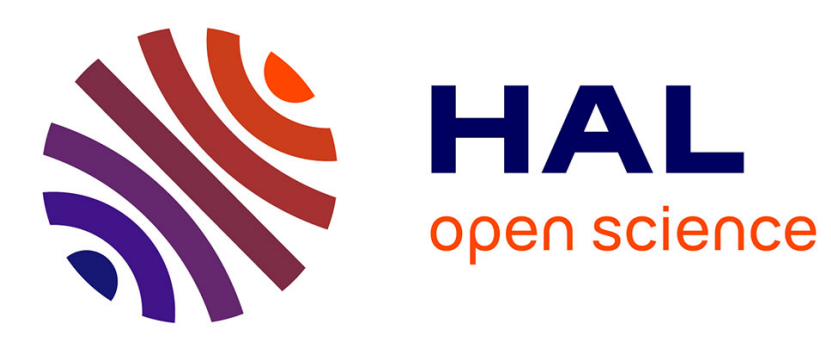

\title{
Opacity Enforcing Control Synthesis
}

Jérémy Dubreil, Philippe Darondeau, Hervé Marchand

\section{To cite this version:}

Jérémy Dubreil, Philippe Darondeau, Hervé Marchand. Opacity Enforcing Control Synthesis. Workshop on Discrete Event Systems, WODES'08, May 2008, Goteborg, Sweden. pp.28-35. inria00344269

\section{HAL Id: inria-00344269 \\ https://hal.inria.fr/inria-00344269}

Submitted on 4 Dec 2008

HAL is a multi-disciplinary open access archive for the deposit and dissemination of scientific research documents, whether they are published or not. The documents may come from teaching and research institutions in France or abroad, or from public or private research centers.
L'archive ouverte pluridisciplinaire HAL, est destinée au dépôt et à la diffusion de documents scientifiques de niveau recherche, publiés ou non, émanant des établissements d'enseignement et de recherche français ou étrangers, des laboratoires publics ou privés. 


\title{
Opacity Enforcing Control Synthesis
}

\author{
Jérémy Dubreil, Philippe Darondeau and Hervé Marchand \\ INRIA Rennes - Bretagne Atlantique, Rennes, France \\ e-mail : \{First.Last $\} @$ irisa.fr
}

\begin{abstract}
Given a finite transition system and a regular predicate, we address the problem of computing a controller enforcing the opacity of the predicate against an attacker (that partially observes the system), supposedly trying to push the system to reveal the predicate. Assuming that the controller can only control a subset of the events it observes (possibly different from the ones of the attacker), we show that an optimal control always exists and provide sufficient conditions under which it is regular and effectively computable. These conditions rely on the inclusion relationships between the observable alphabets of the attacker and the controller and the controllable alphabet.

Index Terms - control, security, opacity, discrete event systems, partial observation
\end{abstract}

\section{INTRODUCTION}

Opacity, whose goal is to oppose diagnosis, was introduced in [1] and [2]. Given a system, equipped with a map sending (prefixes of) executions to observations, an opaque predicate is a set of executions such that every execution in the set is observationally equivalent to some execution outside the set. So, membership to an opaque predicate is never disclosed by observation. Anonymity and non-interference may be reduced to the opacity of suitable predicates for suitable observation maps [2]. In this paper, we concentrate on finite transition systems labelled over an alphabet $\Sigma$, on predicates defined by regular sets of execution traces in $\Sigma^{*}$, and on observation maps induced by the projection of execution traces on a sub-alphabet $\Sigma_{a}$ of $\Sigma$, modeling the attacker's alphabet. Under these assumptions, opacity can be decided although it cannot be expressed in the modal $\mu$-calculus [3].

We are specially interested in cases when the predicate of interest is non-opaque, i.e. the system leaks confidential information. A possible arrangement is then to augment the system with a monitor, responsible for detecting when confidential information was leaked or will be leaked unless one halts the system immediately. Assuming that monitors observe only a subset $\Sigma_{m}$ of the events of the system, which needs not be a subset of $\Sigma_{a}$, necessary and sufficient conditions for the existence of monitors were obtained in [4]. We want to take one step further by providing a controller that does enforce the opacity of the predicate by disabling at each stage (of an execution) the least subset of events such that confidential information is not leaked sooner or later. Assuming that controllers observe all events and all events can be controlled, sufficient conditions for the existence of finite state controllers were proposed in [5] (the opacity of several predicates is enforced there on concurrent attackers). We consider here one predicate and one attacker, but we relax the assumptions on controllable and observable events. Namely, if $\Sigma$ is the set of events of the system, let $\Sigma_{a} \subseteq \Sigma$ be the attacker's alphabet, and let $\Sigma_{c}$ and $\Sigma_{m}$ be the subsets of events controlled or observed by the controller, respectively, then we assume that $\Sigma_{c} \subseteq \Sigma_{m}$ and $\Sigma_{a}$ compares both with $\Sigma_{c}$ and $\Sigma_{m}$.

Let $L(G) \subseteq \Sigma^{*}$ be the regular language of the system $G$ and let $L_{\varphi} \subseteq \Sigma^{*}$ be the regular but non-opaque predicate whose opacity should be enforced by control. Not taking into account controllability and observability, there is a largest subset $L_{1}$ of $L(G)$ such that $L_{\varphi}$ is opaque w.r.t. $L_{1}$ and $\Sigma_{a}$, and $L_{1}$ is regular [5]. As $\Sigma_{c} \subseteq \Sigma_{m}$, there exists a most permissive controller $K_{1}$ confining the system to $L_{1}$ and $K_{1}$ is regular. Unfortunately, this controller does not always enforce the opacity of $L_{\varphi}$ (unless $\Sigma_{a} \subseteq \Sigma_{c}$ or $\Sigma_{m} \subseteq \Sigma_{a}$ as we shall explain later on). The reason why it fails to do so is that a complete description of the closed-loop system may be available to the attacker and new confidential information on the execution may be inferred from this knowledge. To solve the problem, one might think of iterating the construction, thus producing a decreasing chain of regular languages $L(G)=K_{0} \supseteq K_{1} \supseteq K_{2} \supseteq \ldots$. Unfortunately, the iteration may be infinite, hence it may not yield an effective construction of $\cap_{i} K_{i}$ and it does not show either that this limit is regular.

Our contribution is twofold. For the cases $\Sigma_{a} \subseteq \Sigma_{c}$ and $\Sigma_{m} \subseteq \Sigma_{a}$, we show that the optimal opacity control can be computed within the framework of Ramadge and Wonham's theory. For the remaining case $\Sigma_{c} \subseteq \Sigma_{a} \subseteq$ $\Sigma_{m}{ }^{1}$, for which the iteration may be infinite, we supply an alternative algorithm that computes the limit of the infinite iteration described above. The algorithm works in double exponential time. We do not investigate optimizations nor heuristics in this paper for our primary goal is to show that the construction of the optimal opacity control is effective.

This work has loose relationship with the earlier work done by Schneider on security automata [6], subsequently extended to edit automata [7]. The goal pursued in [6] was to produce interface automata that enforce security policies $L_{\varphi}$, meaning that the interface automaton rejects those inputs from the environment that would lead the system to leave the subset of safe execution prefixes $L_{\varphi}$. In our case, the role of the controller is not to confine the executions of the system 
to $L_{\varphi}$ but to the largest opaque subset of $L(G)$ w.r.t. $L_{\varphi}$ and $\Sigma_{a}$. On the other hand, whenever $\Sigma_{c} \subseteq \Sigma_{a}$, our controllers may be seen as interface automata, as they reject events from the attacker's alphabet exclusively.

The rest of the paper is organized as follows. Section 2 fixes some notation. Section 3 brings back the basics of opacity properties and it sets the opacity control problem. Section 4 brings back the theory of Supervisory Control. Section 5, which is the core of the paper, contains our contribution. Optimal opacity control is obtained whenever $\Sigma_{c} \subseteq \Sigma_{m}$ and $\Sigma_{a}$ compares with both of them. Moreover, we produce an example showing that the problem cannot be solved in the framework of Ramadge and Wonham's theory when $\Sigma_{c} \subseteq \Sigma_{a} \subseteq \Sigma_{m}$. Section 6 is a brief conclusion pointing to open problems.

\section{NOTATIONS}

Let $\Sigma$ be a finite alphabet of events. A string is a finite sequence of events. The set of all strings is denoted by $\Sigma^{*}$. Any subset of $\Sigma^{*}$ is called a language over $\Sigma$. Let $L$ be a language over $\Sigma$. The prefix-closure of $L$ is defined as $\bar{L}=\left\{s \in \Sigma^{*} \mid \exists t \in \Sigma^{*}\right.$ s.t. $\left.s t \in L\right\}$. We assume that systems are Labelled Transitions Systems (LTS) as follows.

Definition 1 (LTS): An LTS over $\Sigma$ is a 4-tuple $G=$ $\left(Q_{G}, \Sigma, \delta_{G}, q_{0}^{G}\right)$ where $Q_{G}$ is a finite set of states, $\Sigma$ is the finite set of events of $G, q_{0}^{G} \in Q_{G}$ is the initial state, and $\delta_{G}: Q_{G} \times \Sigma \rightarrow Q_{G}$ is a partial transition function. $\diamond$ In the sequel, we write $q \stackrel{a}{\rightarrow}_{G} q^{\prime}$ if $\delta(q, a)=q^{\prime}$ and $q \stackrel{a}{\rightarrow}_{G}$ if $\exists q^{\prime} \in Q_{G}, q \stackrel{a}{\rightarrow}_{G} q^{\prime}$. We extend $\rightarrow_{\mathrm{G}}$ to arbitrary sequences by setting $q \stackrel{\varepsilon}{\rightarrow}$ G $q$ for all states $q$, and $q \stackrel{s \sigma}{\rightarrow}_{\mathrm{G}} q^{\prime}$ whenever $q \stackrel{s}{\rightarrow}_{\mathrm{G}} q^{\prime \prime}$ and $q^{\prime \prime} \stackrel{\sigma}{\rightarrow}_{\mathrm{G}} q^{\prime}$, for some $q^{\prime \prime} \in Q_{\mathrm{G}}, s \in \Sigma^{*}$ and $\sigma \in \Sigma$. We denote

$$
\mathcal{T}_{G}=\left\{\left(q, \sigma, q^{\prime}\right) \in Q \times \Sigma \times Q: q \stackrel{\sigma}{\rightarrow} q^{\prime}\right\}
$$

the set of transitions of $G$ and $L(G)=\left\{l \in \Sigma^{*} \mid q_{0}^{\mathrm{G}} \stackrel{l}{\rightarrow}_{G}\right\}$ the set of its execution traces. Given non-empty subsets $I_{\mathrm{G}}, F_{\mathrm{G}} \subseteq$ $Q_{G}$, the definitions extend to $L_{F_{G}}(G)=\left\{s \in \Sigma^{*} \mid \exists q \in\right.$ $\left.F_{G}, q_{0}^{\mathrm{G}} \stackrel{s}{\rightarrow}_{G} q\right\}$ (the set of execution traces ending in a final state of $\left.F_{G}\right)$ and $L_{I_{G}, F_{G}}(G)=\left\{s \in \Sigma^{*} \mid \exists q^{\prime} \in I_{G}, \exists q \in\right.$ $\left.F_{G}, q^{\prime} \stackrel{s}{\rightarrow}_{G} q\right\}$ (the set of partial execution traces starting in a state of $I_{G}$ and ending in a state of $F_{G}$ ).

Opacity control aims at preventing an attacker $\mathcal{A}$ from deducing confidential information on the execution of a system from the observation of a subset of events $\Sigma_{a}$. To model this, we use the classical notion of projection. We simply denote by $P_{\Sigma_{a}}$ the projection from $\Sigma^{*}$ to $\Sigma_{a}^{*}$ that erases in a sequence of $\Sigma^{*}$ all events not in $\Sigma_{a}$. This definition extends to (regular) languages: $P_{\Sigma_{a}}(K)=\left\{\mu \in \Sigma_{a}^{*} \mid \exists s \in K, \mu=\right.$ $\left.P_{\Sigma_{a}}(s)\right\}$. Conversely, given $K \subseteq \Sigma_{a}^{*}$, the inverse projection of $K$ is $P_{\Sigma_{a}}^{-1}(K)=\left\{s \in \Sigma^{*} \mid P_{\Sigma_{a}}(s) \in K\right\}$. Given an LTS $G$ over $\Sigma$ and a set of observable events $\Sigma_{a} \subseteq \Sigma$, the set of observed traces of $G$ is $P_{\Sigma_{a}}(L(G))$. Given two sequences $s, s^{\prime} \in \Sigma^{*}$, we let $s \sim_{a} s^{\prime}$ in case $P_{\Sigma_{a}}(s)=P_{\Sigma_{a}}\left(s^{\prime}\right)$ and denote $[s]_{a}=P_{\Sigma_{a}}^{-1}\left(P_{\Sigma_{a}}(s)\right)$ the equivalence class of $s$.

Lemma 1: Let $\Sigma_{a} \subseteq \Sigma_{b} \subseteq \Sigma$, then $s \sim_{b} s^{\prime} \Rightarrow s \sim_{a} s^{\prime}$.

\section{THE BASICS OF OPACITY}

Consider an LTS $G$ over $\Sigma$, a regular predicate $L_{\varphi} \subseteq \Sigma^{*}$, and a sub-alphabet $\Sigma_{a} \subseteq \Sigma$. The alphabet $\Sigma_{a}$ defines the interface provided to the user for interacting with $G$. The predicate $L_{\varphi}$ represents a confidential information on the execution of $G$, i.e. if the current trace of execution is $s \in \Sigma^{*}$, the user should not be able to deduce from $P_{\Sigma_{a}}(s)$ and $G$ that $s \in L_{\varphi}$. In this setting, the user is considered as an attacker $(\mathcal{A})$ willing to catch the confidential information and armed for this with full information on the structure of $G$ but only partial information upon its behavior, namely the observed trace in $\Sigma_{a}^{*}$. In order that the confidential information is never leaked, it is necessary and sufficient that $L_{\varphi}$ is an opaque predicate according to the following definition, adapted from [2].

Definition 2 (Opacity): $L_{\varphi}$ is said to be opaque w.r.t. $L(G)$ and $\Sigma_{a}$ if

$$
\forall s \in L(G), \quad[s]_{a} \cap L(G) \not L_{\varphi}
$$

In other words, $L_{\varphi}$ is opaque w.r.t. $L(G)$ and $\Sigma_{a}$ if and only if $\forall \mu \in P_{\Sigma_{a}}(L(G)), P_{\Sigma_{a}}^{-1}(\mu) \cap L(G) \nsubseteq L_{\varphi}$, and $L_{\varphi}$ is non-opaque w.r.t. $L(G)$ and $\Sigma_{a}$ if and only if $\exists \mu \in$ $P_{\Sigma_{a}}(L(G)), P_{\Sigma_{a}}^{-1}(\mu) \cap L(G) \subseteq L_{\varphi}$.

Example 1: Consider the two specifications $G_{1}$ and $G_{2}$ of a coffee-machine depicted in Figures 1 and 2, and let $\Sigma_{a}=\{$ coinIn, coinOut, cancel, confirm, coffeeOut $\}$. Consider the predicate $L_{\varphi}=\Sigma^{*}$.full. $\Sigma^{*}$. Then, $L_{\varphi}$ is not

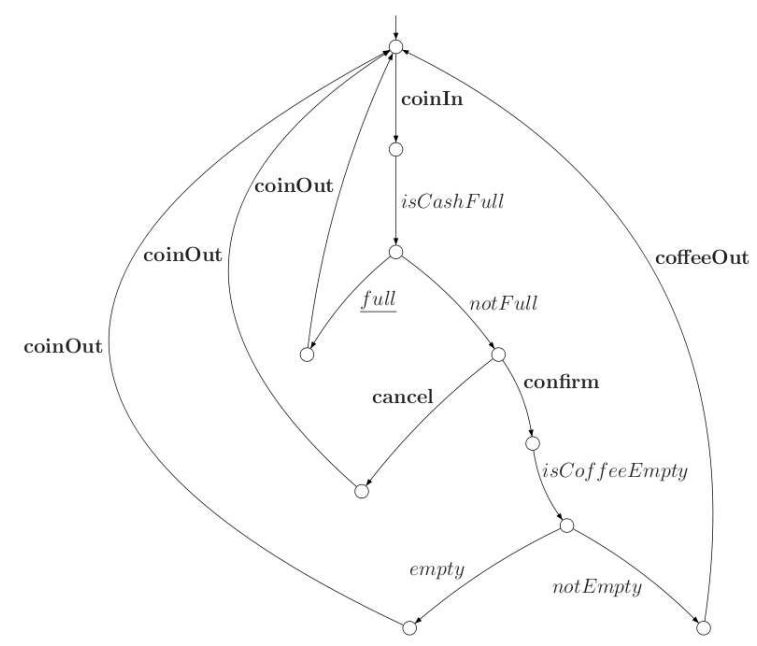

Fig. 1. The predicate $L_{\varphi}$ is non opaque w.r.t. $G_{1}$ and $\Sigma_{a}$

opaque with respect to $L\left(G_{1}\right)$ and $\Sigma_{a}$, since e.g. for the observed trace coinIn.coinOut for which the only possible execution trace is coinIn.isCashFull.full.coinOut $\in L_{\varphi}$. A contrario $L_{\varphi}$ is opaque with respect to $L\left(G_{2}\right)$ and $\Sigma_{a}$.

If $L_{\varphi}$ is not opaque w.r.t. $L(G)$ and $\Sigma_{a}$, then it is still possible to restrict the behavior of $G$ so that $L_{\varphi}$ becomes opaque. This can be obtained by withdrawing from $L(G)$ all words $u . v$ such that $u \sim_{a} u^{\prime} \Rightarrow u^{\prime} \in L_{\varphi}$ for all $u^{\prime} \in L(G)$.

Proposition 1 ([5]): Given a system $G$ and a predicate $L_{\varphi}$, there exists a supremal prefix-closed sub-language of 


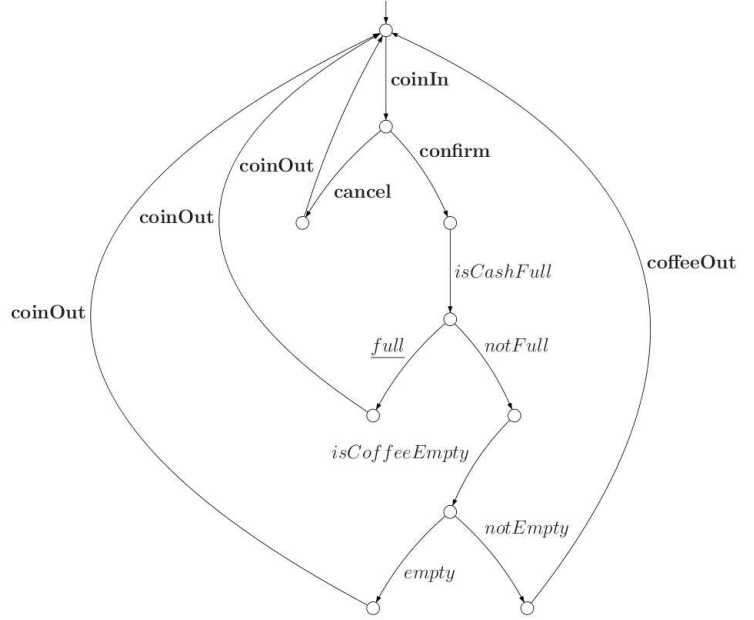

Fig. 2. The predicate $L_{\varphi}$ is opaque w.r.t. $G_{2}$ and $\Sigma_{a}$

$L(G)$, noted $\mathrm{OP}^{\uparrow}\left(L(G), L_{\varphi}, \Sigma_{a}\right)$, such that $L_{\varphi}$ is opaque w.r.t. $\mathrm{OP}^{\uparrow}\left(L(G), L_{\varphi}, \Sigma_{a}\right)$ and $\Sigma_{a}$, and it is given by

$$
\begin{aligned}
& \mathrm{OP}^{\uparrow}\left(L(G), L_{\varphi}, \Sigma_{a}\right)= \\
& L(G) \backslash\left(\left(L(G) \backslash P_{\Sigma_{a}}^{-1}\left(P_{\Sigma_{a}}\left(L(G) \backslash L_{\varphi}\right)\right)\right) . \Sigma^{*}\right)
\end{aligned}
$$

Intuitively, the language $P_{\Sigma_{a}}^{-1}\left(P_{\Sigma_{a}}\left(L(G) \backslash L_{\varphi}\right)\right)$ is the set of the "safe" sequences that do not reveal $L_{\varphi}{ }^{2}$, whereas any sequence in $L(G) \backslash P_{\Sigma_{a}}^{-1}\left(P_{\Sigma_{a}}\left(L(G) \backslash L_{\varphi}\right)\right)$ reveals $L_{\varphi}$ (these sequences are extended with $\Sigma^{*}$ because, once $L_{\varphi}$ has been revealed, this holds for ever).

It follows from proposition 1 that $\operatorname{OP}^{\uparrow}\left(L(G), L_{\varphi}, \Sigma_{a}\right)$ is the union of all prefix-closed sub-languages $L^{\prime}$ of $L(G)$, such that $L_{\varphi}$ is opaque w.r.t. $L^{\prime}$ and $\Sigma_{a}$ [5]. Therefore, $\mathrm{OP}^{\uparrow}$ is monotone in the first argument. Note that $\mathrm{OP}^{\uparrow}\left(L(G), L_{\varphi}, \Sigma_{a}\right)$ can be empty. In that case, there is no way to enforce opacity by restricting the behavior of the system.

Remark 1: If $L_{\varphi}$ is opaque w.r.t. $L_{1}$ and $L_{2}$, then it is opaque w.r.t. $L_{1} \cup L_{2}$, but not necessarily w.r.t. $L_{1} \cap L_{2}$. Similarly, if $L_{1} \subseteq L \subseteq L_{2}, L_{\varphi}$ may be opaque w.r.t. $L$ but not opaque w.r.t. $L_{1}$ or $L_{2}$.

Next, we establish a helpful lemma, stating that if a sequence $s$ belongs to $\mathrm{OP}^{\uparrow}\left(L(G), L_{\varphi}, \Sigma_{a}\right)$, then any sequence in $L(G)$ observationally equivalent to $s$ also belongs to $\mathrm{OP}^{\uparrow}\left(L(G), L_{\varphi}, \Sigma_{a}\right)$.

Lemma 2: $\forall s \in \mathrm{OP}^{\uparrow}\left(L(G), L_{\varphi}, \Sigma_{a}\right)$,

$$
[s]_{a} \cap L(G) \subseteq \mathrm{OP}^{\uparrow}\left(L(G), L_{\varphi}, \Sigma_{a}\right)
$$

Proof: Let $s^{\prime} \in[s]_{a} \cap L(G)$, then by definition $s \sim_{a} s^{\prime}$ and $s^{\prime} \in L(G)$. Suppose for a contradiction that $s^{\prime} \notin \operatorname{OP}^{\uparrow}\left(L(G), L_{\varphi}, \Sigma_{a}\right)$, then $s^{\prime}=u v$ for some $u$ such that $u \sim_{a} u^{\prime} \Rightarrow u^{\prime} \in L_{\varphi}$ for all $u^{\prime} \in L(G)$. As $s \sim_{a} s^{\prime}$, $s=u$ " $v "$ for some $u " \in L(G)$ such that $u \sim_{a} u$ ". Therefore, $u " \sim_{a} u^{\prime} \Rightarrow u^{\prime} \in L_{\varphi}$ for all $u^{\prime} \in L(G)$, showing that $s \notin \mathrm{OP}^{\uparrow}\left(L(G), L_{\varphi}, \Sigma_{a}\right)$, a contradiction.

Dually, this lemma implies that if a sequence $s$ belongs to $L(G) \backslash \mathrm{OP}^{\uparrow}\left(L_{\varphi}, L(G), \Sigma_{a}\right)$, then no sequence observationally

\footnotetext{
${ }^{2}$ Note that this language is not prefix-closed.
}

equivalent to $s$ belongs to $\operatorname{OP}^{\uparrow}\left(L(G), L_{\varphi}, \Sigma_{a}\right)$. In other words, when computing the supremal sub-language of $L(G)$ with respect to which $L_{\varphi}$ is opaque, each equivalence class of $L(G)$ w.r.t. $\Sigma_{a}$ is either entirely kept or removed.

Our goal is to enforce opacity by supervisory control, which puts strong conditions on the admissible restrictions of $L(G)$ (due to the so-called controllability and observability conditions that a controller has to fulfill to be implementable). We will also compute the most permissive opacity control in the form of a regular sub-language of $L(G)$. Next section brings back a few notions of supervisory control theory.

\section{The BASICS OF SUPERVISORY CONTROL}

Given a prefix-closed behavior $K \subseteq L(G) \subseteq \Sigma^{*}$ expected from the system $G$, the goal of supervisory control is to enforce this behavior on $G$ by pairing this system with a monitor (also called controller) that observes a subset $\Sigma_{m}$ of the events in $\Sigma$ and controls a subset $\Sigma_{c}$ of the events in $\Sigma$, i.e. enables or disables each instance of these controllable events. $\Sigma \backslash \Sigma_{c}$ is the set of uncontrollable events. $\Sigma \backslash \Sigma_{m}$ is the set of unobservable events. We now recall some basic concepts of supervisory control theory. More information on the computational aspects can be found in [8].

Definition 3: A prefix-closed language $K \subseteq L(G)$ is controllable w.r.t. $L(G)$ and $\Sigma_{c}$ if $K .\left(\Sigma \backslash \Sigma_{c}\right) \cap L(G) \subseteq K$. This definition states that if $K$ is controllable, then no uncontrollable events need to be disabled to exactly confine the system $L(G)$ to $K$. Note that the union of an arbitrary number of controllable languages is controllable.

Definition 4: Assuming that $\Sigma_{c} \subseteq \Sigma_{m}$, a prefixclosed language $K$ is observable w.r.t. $L(G)$ and $\Sigma_{m}$ if $P_{\Sigma_{m}}^{-1}\left[P_{\Sigma_{m}}(K)\right] \cap L(G) \subseteq K^{3}$.

Intuitively, $K$ is observable, if $K$ can be exactly recovered from its projection $P_{\Sigma_{m}}(K)$ and $L(G)$. Note that this is a necessary condition for a controller that forces the system to behave like $K$ to be implementable. In other words, from a control point of view, when disabling an event $c$ after the execution of $s$, then $c$ has to be disabled after all execution traces of $[s]_{m}$. Under the assumption $\Sigma_{c} \subseteq \Sigma_{m}$, the union of an arbitrary number of observable languages is observable. Therefore, under this assumption, both controllability and observability are stable under union of languages, and there exists a supremal controllable and observable prefix-closed sub-language of $K$, that we denote

$$
\mathrm{CO}^{\uparrow}\left(K, L(G), \Sigma_{c}, \Sigma_{m}\right)
$$

The language $\mathrm{CO}^{\uparrow}\left(K, L(G), \Sigma_{c}, \Sigma_{m}\right)$ represents the largest behavior included in $K(\subseteq L(G))$ that can be enforced by control. Moreover, $\mathrm{CO}^{\uparrow}$ is monotone in the first argument.

Lemma 3: Assuming that $\Sigma_{c} \subseteq \Sigma_{m}$, let $s \in K \backslash$ $\mathrm{CO}^{\uparrow}\left(K, L(G), \Sigma_{c}, \Sigma_{m}\right)$, then

$$
[s]_{m} \cap \mathrm{CO}^{\uparrow}\left(K, L(G), \Sigma_{c}, \Sigma_{m}\right)=\emptyset
$$

\footnotetext{
${ }^{3}$ Note that we have given here the formal definition of normality. Under the assumption $\Sigma_{c} \subseteq \Sigma_{m}$, observability and normality coincide [9].
} 
Proof: Because $\mathrm{CO}^{\uparrow}\left(K, L(G), \Sigma_{c}, \Sigma_{m}\right)$ is observable, this set and its relative complement are unions of equivalence classes of $\sim_{m}$.

Similarly to lemma 2 , the equivalence classes of $L(G)$ w.r.t. $\Sigma_{m}$ are preserved by control.

\section{ENFORCING OPACITY BY CONTROL}

Our purpose is to solve the opacity control problem stated as follows.

Problem: Show that the set of controllable and observable restrictions (i.e. sub-languages) of $L(G)$ enforcing the opacity of $L_{\varphi}$ either is empty or has a greatest element and compute this maximal permissive controllable and observable sub-language of $L(G)$.

In the sequel, we shall assume that an attacker has a full knowledge of the structure of $G$, knows the interface of the controller $\Sigma_{m}$ and is able to perform in his head all calculations that the administrator has made to compute this controller. In particular, this entails that the structure of the controlled system may be available to the attacker, thus possibly inducing new confidential information flow. This assumptions are at present informal, but might be formalized e.g. using language theory and epistemic logic. Moreover, in the rest of the paper, it is always assumed that $\Sigma_{c} \subseteq \Sigma_{m}$ (the controllable events are observed by the controller).

\section{A. Characterization of the solution}

We now investigate the existence of a supremal solution to the opacity control problem. To do so, we consider the set

$$
\begin{aligned}
\mathcal{C}_{\varphi}=\{L \subseteq L(G) \mid & L_{\varphi} \text { is opaque w.r.t. } L \text { and } \Sigma_{a}, \\
& L \text { is prefix-closed, } \\
& L \text { is controllable w.r.t. } L(G) \text { and } \Sigma_{c}, \\
& \left.L \text { is observable w.r.t. } L(G) \text { and } \Sigma_{m}\right\}
\end{aligned}
$$

and the prefix-closed language

$$
\operatorname{CO-OP}^{\uparrow}\left(L(G), L_{\varphi}, \Sigma_{a}, \Sigma_{m}, \Sigma_{c}\right)=\bigcup_{L \in \mathcal{C}_{\varphi}} L
$$

Proposition 2: If $\mathrm{CO}-\mathrm{OP}^{\uparrow}\left(L(G), L_{\varphi}, \Sigma_{a}, \Sigma_{m}, \Sigma_{c}\right) \neq \emptyset$, then it is the supremal sub-language of $L(G)$ such that

(1) ${\mathrm{CO}-O P^{\uparrow}}\left(L(G), L_{\varphi}, \Sigma_{a}, \Sigma_{m}, \Sigma_{c}\right)$ is controllable and observable w.r.t. $L(G), \Sigma_{c}$ and $\Sigma_{m}$,

$$
\begin{aligned}
& \text { and } L_{\varphi} \quad \text { is } \quad \text { opaque } \\
& \operatorname{CO-OP}^{\uparrow}\left(L(G), L_{\varphi}, \Sigma_{a}, \Sigma_{m}, \Sigma_{c}\right) \text { and } \Sigma_{a} .
\end{aligned}
$$

Otherwise, no control can enforce the opacity of $L_{\varphi}$.

Proof: If CO-OP ${ }^{\uparrow}\left(L(G), L_{\varphi}, \Sigma_{a}, \Sigma_{m}, \Sigma_{c}\right) \neq \emptyset$, then it is the union of an arbitrary number of languages that are controllable, observable and such that $L_{\varphi}$ is opaque w.r.t. the corresponding restrictions of $L(G)$. These three properties are stable under arbitrary union of languages (under the hypothesis that $\left.\Sigma_{c} \subseteq \Sigma_{m}\right)$. So $\mathrm{CO}-O P^{\uparrow}\left(L(G), L_{\varphi}, \Sigma_{a}, \Sigma_{m}, \Sigma_{c}\right)$ satisfies (1) and (2).

Even though the previous proposition entails the existence of a unique maximal sub-language of $L(G)$, that is controllable, observable and in restriction to which $L_{\varphi}$ is opaque, we still have to examine whether this language is regular (or at least, to exhibit sufficient conditions for regularity) and to provide an effective computation of this language.

It may be remarked that restricting languages to ensure controllability and observability does not always preserve opacity and the other way round (See Example 2). Thus, in a first attempt towards an effective computation of CO-OP ${ }^{\uparrow}\left(L(G), L_{\varphi}, \Sigma_{a}, \Sigma_{m}, \Sigma_{c}\right)$, following the classical methodology of Supervisory Control Theory ${ }^{4}$, we establish below a fix-point characterization of this language by alternating the computation of the supremal sub-language that ensures the opacity of $L_{\varphi}$ and the supremal controllable and observable sub-language.

Consider the operator

$$
\mathcal{K}(\bullet)=\mathrm{CO}^{\uparrow}\left(\mathrm{OP}^{\uparrow}\left(\bullet, L_{\varphi}, \Sigma_{a}\right), L(G), \Sigma_{c}, \Sigma_{m}\right) .
$$

Remark that $\mathcal{K}(\bullet)$ is monotone w.r.t. set inclusion. Now, as the prefix-closed subsets of $L(G)$ form a complete sub-lattice of $\mathcal{P}\left(\Sigma^{*}\right)$, it follows from Knaster-Tarski's Theorem [10] that $\mathcal{K}(\bullet)$ has a greatest fix-point in this sub-lattice. Let $K\left(L(G), L_{\varphi}\right)$ be the greatest fix-point of the operator $\mathcal{K}(\bullet)$ included in $L(G)^{5}$.

Proposition 3:

$$
\begin{aligned}
& K\left(L(G), L_{\varphi}\right)={\mathrm{CO}-O{ }^{\uparrow}}^{\uparrow}\left(L(G), L_{\varphi}, \Sigma_{a}, \Sigma_{m}, \Sigma_{c}\right) \\
& \text { Proof: } \quad \text { We denote } L^{c}
\end{aligned}
$$

${\mathrm{CO}-O P^{\uparrow}}^{\uparrow}\left(L(G), L_{\varphi}, \Sigma_{a}, \Sigma_{m}, \Sigma_{c}\right)$. Clearly, $L_{\varphi}$ is opaque w.r.t. $K\left(L(G), L_{\varphi}\right)$ and $\Sigma_{a}$. This language is controllable and observable, hence $K\left(L(G), L_{\varphi}\right) \subseteq L^{c}$.

Moreover, we have $L^{c} \subseteq L(G)=\mathcal{K}^{0}(L(G))$. Assume now that $L^{c} \subseteq \mathcal{K}^{i}(L(G))$ for some $i$. Then, from the monotony of $\mathcal{K}(\bullet)$, we get $\mathcal{K}^{i+1}(L(G)) \supseteq \mathcal{K}\left(L^{c}\right)=L^{c}$, since $L^{c}$ controllable and observable and $L_{\varphi}$ is opaque w.r.t. $L^{c}$ and $\Sigma_{a}$. By transfinite induction, it follows that $L^{c} \subseteq \mathcal{K}^{\alpha}(L(G))$ for every ordinal $\alpha$. Therefore $L^{c} \subseteq$ $\cap_{\alpha} \mathcal{K}^{\alpha}(L(G))=K\left(L(G), L_{\varphi}\right)$.

Note that this fix-point characterization of ${\mathrm{CO}-O P^{\uparrow}}^{\uparrow}\left(L(G), L_{\varphi}, \Sigma_{a}, \Sigma_{m}, \Sigma_{c}\right)$ does not ensure that this language can be always computed by a finite iteration as the following example shows.

Example 2: Consider the LTS $G$ shown in Fig. 3 where $\Sigma_{a}=\{A, B, c\}, \Sigma_{m}=\Sigma, \Sigma_{c}=\{c\}$ and the predicate $L_{\varphi}$ is the set of the sequences that reach the states represented with squares in $G$. Let $K_{i}=\mathcal{K}^{i}(L(G))$ denote the language computed after $i$ iterations of the operator $\mathcal{K}(\bullet)$.

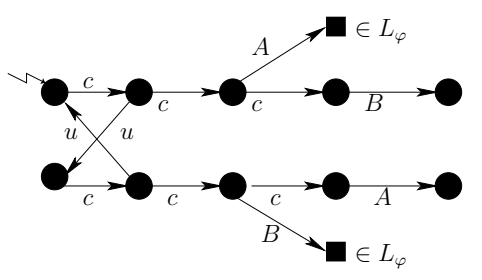

Fig. 3. $L(G)$ and $L_{\varphi}$

In $L(G)$, the sole string that belongs to $L_{\varphi}$, and therefore reveals it, is c.c.A, which requires to disable the second event

\footnotetext{
${ }^{4}$ that ensures both non-blocking and controllability

${ }^{5} K\left(L(G), L_{\varphi}\right)$ is also the greatest fix-point of the operator $\mathcal{K}^{\prime}=$ $\mathrm{OP}^{\uparrow}\left(\mathrm{CO}^{\uparrow}\left(\bullet, L(G), \Sigma_{c}, \Sigma_{m}\right), L_{\varphi}, \Sigma_{a}\right)$.
} 
$c$, seeing that $A$ is uncontrollable. The LTS that generates $K_{1}$ is represented in Fig. 4(a). In $K_{1}$, c.c. A has disappeared and

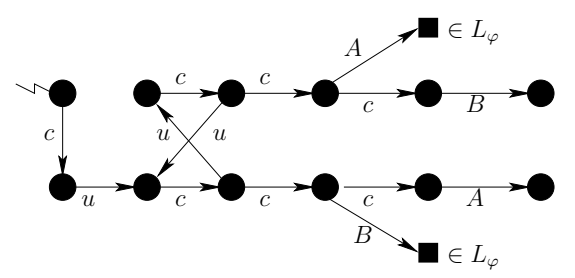

(a)

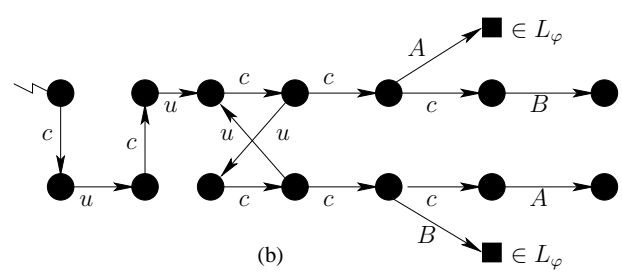

Fig. 4. $K_{1}$ and $K_{2}$

the sole string that belongs to $L_{\varphi}$, and therefore reveals it, is c.u.c.c.B, which requires to disable the event c after c.u.c. The result $\left(K_{2}\right)$ is depicted in Fig. 4(b). After $2 i$ iterations of the operator $\mathcal{K}(\bullet)$, one gets the language $K_{2 i}$ generated by the LTS depicted in Fig. 5(a). In $K_{2 i}$, the string (c.u $)^{2 i}$.c.c.A

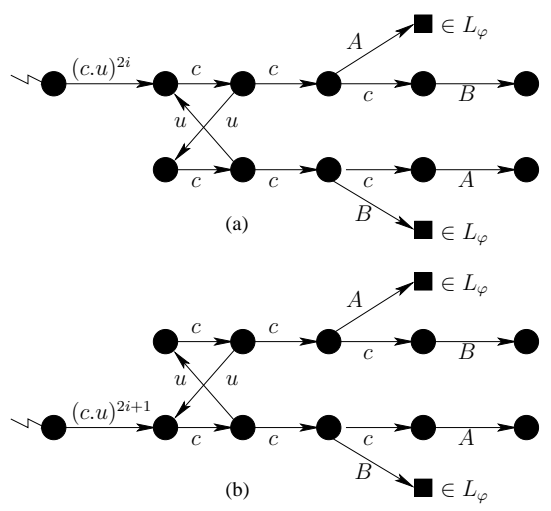

Fig. 5. $K_{2 i}$ and $K_{2 i+1}$

reveals $L_{\varphi}$ and it must be eliminated by disabling the last $c$, which is done in the language $K_{2 i+1}$ (See Fig. 5(b). But, in $K_{2 i+1}$, the string $(\text { c.u })^{2 i+1}$.c.c.B reveals $L_{\varphi}$. Disabling the last c leads to $K_{2 i+2}$, which reproduces the situation found in $K_{2 i}$ (up to replacing the prefix $(\text { c.u })^{2 i}$ by $(c . u)^{2 i+2}$. Finally, even though the limit $K\left(L(G), L_{\varphi}\right)$ of this decreasing chain is the regular language (c.u.c.u $)^{*}$, the fix-point iteration produces a strictly decreasing and infinite sequence of languages $K_{j}$ showing that the above algorithm may not terminate. $\diamond$ In the rest of the paper, we investigate sufficient conditions, induced by relations between the alphabets $\Sigma_{c}, \Sigma_{a}$ and $\Sigma_{m}$, under which $\mathrm{CO}-\mathrm{OP}^{\uparrow}\left(L(G), L_{\varphi}, \Sigma_{a}, \Sigma_{m}, \Sigma_{c}\right)$ is regular and one can effectively compute uniformly from the arguments of $\mathrm{CO}-\mathrm{OP}^{\uparrow}(\bullet)$ a finite automaton generating this optimal opacity control.

But first, we establish a proposition that helps to simplify the remaining proofs (In Sections V-B.2 and V-B.3). This proposition states that whenever $\Sigma_{a} \subseteq \Sigma_{m} \subseteq \Sigma$, we can reformulate the control problem in terms of the observed system w.r.t. $\Sigma_{m}$ and a new predicate $L_{\varphi}^{m} \subseteq \Sigma_{m}^{*}$ derived from $L_{\varphi}$ and $\Sigma_{m}$, solve the problem in this new setting (thus, under full observation) and lift up the solution to the initial setting. The intuition is that observing events from $\Sigma \backslash \Sigma_{m}$ could not help the controller anyway.

Proposition 4: Assume that $\Sigma_{a} \subseteq \Sigma_{m} \subseteq \Sigma$ and $\Sigma_{c} \subseteq$ $\Sigma_{m}$. Let $L_{\varphi}, L(G) \subseteq \Sigma^{*}$, then

$$
\begin{aligned}
& \operatorname{CO-OP}^{\uparrow}\left(L(G), L_{\varphi}, \Sigma_{a}, \Sigma_{m}, \Sigma_{c}\right)= \\
& \quad P_{\Sigma_{m}}^{-1}\left(\operatorname{CO}-\mathrm{OP}^{\uparrow}\left(P_{\Sigma_{m}}(L(G)), L_{\varphi}^{m}, \Sigma_{a}, \Sigma_{m}, \Sigma_{c}\right)\right) \cap L(G)
\end{aligned}
$$

where $L_{\varphi}^{m}=\left\{\rho \in P_{\Sigma_{m}}(L(G)): P_{\Sigma_{m}}^{-1}(\rho) \cap L(G) \subseteq L_{\varphi}\right\}$

Proof: Consider the following languages:

- $K=\mathrm{CO}-O P^{\uparrow}\left(L(G), L_{\varphi}, \Sigma_{a}, \Sigma_{m}, \Sigma_{c}\right)$,

- $F={\mathrm{CO}-O P^{\uparrow}}^{\uparrow}\left(P_{\Sigma_{m}}(L(G)), L_{\varphi}^{m}, \Sigma_{a}, \Sigma_{m}, \Sigma_{c}\right)$

- $H=P_{\Sigma_{m}}^{-1}(F) \cap L(G)$.

We will prove that $H=K$.

Let us first prove that $L_{\varphi}$ is opaque w.r.t. $H$ and $\Sigma_{a}$. Consider $s \in H \cap L_{\varphi}$. As $P_{\Sigma_{m}}(s) \in F$ and $L_{\varphi}^{m}$ is opaque w.r.t. $F$ and $\Sigma_{a}$ (by definition of $F$ ), there exists $\rho \in F$ such that $\rho \sim_{a} P_{\Sigma_{m}}(s)$ and $\rho \in F \backslash L_{\varphi}^{m}$. Then, $\exists s^{\prime} \in$ $P_{\Sigma_{m}}^{-1}(\rho) \cap L(G), s^{\prime} \notin L_{\varphi}$ according to the definition of $L_{\varphi}^{m}$. But $\rho \in F$ implies that $s^{\prime} \in H . P_{\Sigma_{m}}\left(s^{\prime}\right) \sim_{a} P_{\Sigma_{m}}(s)$ and $\Sigma_{a} \subseteq \Sigma_{m}$ implies that $s^{\prime} \sim_{a} s$. So $L_{\varphi}$ is opaque w.r.t. $H$ and $\Sigma_{a}$.

Let us now show that $H$ is controllable. Consider $s \in$ $H, \sigma \in \Sigma \backslash \Sigma_{c}$ such that $s \sigma \in L(G)$. Let $\rho=P_{\Sigma_{m}}(s)$. By definition of $H$, we get $\rho \in F$.

- If $\sigma \notin \Sigma_{m}$ then $P_{\Sigma_{m}}(s \sigma)=\rho$ and finally $s \sigma \in H$.

- If $\sigma \in \Sigma_{m}$, we have $\rho \in F$ and $\rho \sigma \in P_{\Sigma_{m}}(L(G))$. As $F$ is controllable, we get $\rho \sigma \in F$, which entails $s \sigma \in H$ as $s \sigma \in P_{\Sigma_{m}}^{-1}(\{\rho \sigma\})$.

Finally we note that $H$ is observable by construction. As $K$ is the supremal controllable and observable sub-language of $L(G)$ for which $L_{\varphi}$ is opaque, we can conclude that $H \subseteq K$.

Let us now prove that $P_{\Sigma_{m}}(K) \subseteq F$.

- Let $\rho \in P_{\Sigma_{m}}(K)$. There exists $s \in K$ such that $P_{\Sigma_{m}}(s)=\rho$. Since $L_{\varphi}$ is opaque w.r.t. $K$ and $\Sigma_{a}$, there exists $s^{\prime} \in K, s^{\prime} \sim_{a} s$ such that $s^{\prime} \in K \backslash L_{\varphi}$. Let $\rho^{\prime}=P_{\Sigma_{m}}\left(s^{\prime}\right)$. We have $\rho^{\prime} \notin L_{\varphi}^{m}$. As $\rho \sim_{a} \rho^{\prime}$, we conclude that $L_{\varphi}^{m}$ is opaque w.r.t. $P_{\Sigma_{m}}(K)$ and $\Sigma_{a}$.

- Let us show that $P_{\Sigma_{m}}(K)$ is controllable w.r.t. $P_{\Sigma_{m}}(L(G))$ and $\Sigma_{c}$. Let $\rho \in P_{\Sigma_{m}}(K)$ and $\sigma \in \Sigma_{m} \backslash \Sigma_{c}$ such that $\rho \sigma \in P_{\Sigma_{m}}(L(G))$. Then, $\exists s \in K \subseteq L(G)$, such that $P_{\Sigma_{m}}(s)=\rho$ and $s \sigma \in K\left(\Sigma_{m} \backslash \Sigma_{c}\right) \cap L(G)$. Since $K$ is controllable, $s \sigma \in K$ and then $\rho \sigma=$ $P_{\Sigma_{m}}(s \sigma) \in P_{\Sigma_{m}}(K)$. So $P_{\Sigma_{m}}(K)$ is controllable.

Now, $P_{\Sigma_{m}}(K)$ is obviously observable and we get that $P_{\Sigma_{m}}(K) \subseteq F$. This implies that $P_{\Sigma_{m}}^{-1}\left(P_{\Sigma_{m}}(K)\right) \cap L(G) \subseteq$ $H$ and since $K=K \cap L(G) \subseteq P_{\Sigma_{m}}^{-1}\left(P_{\Sigma_{m}}(K)\right) \cap L(G)$, we conclude that $K \subseteq H$ and finally that $H=K$.

\section{B. Effective computation of the supremal solution}

Next, we investigate three sufficient conditions under which $\mathrm{CO}-\mathrm{OP}^{\uparrow}\left(L(G), L_{\varphi}, \Sigma_{a}, \Sigma_{m}, \Sigma_{c}\right)$ is regular and effectively computable.These conditions bear upon the inclusion relationships between the alphabets $\Sigma_{a}, \Sigma_{m}$ and $\Sigma_{c}$. 
1) Assumption 1: $\Sigma_{c} \subseteq \Sigma_{m} \subseteq \Sigma_{a} \subseteq \Sigma$ : Under this assumption, the controller observes and controls only a part of the actions of the attacker, meaning that it is less powerful than the attacker. Nevertheless, this is a sufficient condition allowing to solve the control problem.

Proposition 5: Assume $\Sigma_{c} \subseteq \Sigma_{m} \subseteq \Sigma_{a} \subseteq \Sigma$, then $K_{1}(=\mathcal{K}(L(G)))={\mathrm{CO}-O \mathrm{P}^{\uparrow}}^{\uparrow}\left(L(G), L_{\varphi}, \Sigma_{a}, \Sigma_{m}, \Sigma_{c}\right)$ is regular and effectively computable.

Proof: Let $L_{1}=\mathrm{OP}^{\uparrow}\left(L(G), L_{\varphi}, \Sigma_{a}\right)$, then $K_{1}=$ $\mathrm{CO}^{\uparrow}\left(L_{1}, L(G), \Sigma_{c}, \Sigma_{m}\right)$. Consider $s \in K_{1} \cap L_{\varphi}$. As $L_{\varphi}$ is opaque w.r.t. $L_{1}$ and $\Sigma_{a}, \exists s^{\prime} \in L_{1}$ such that $s \sim_{a} s^{\prime}$ and $s^{\prime} \notin$ $L_{\varphi}$. As $\Sigma_{m} \subseteq \Sigma_{a}$ and $s \sim_{a} s^{\prime}$, we get $s \sim_{m} s^{\prime}$. Hence, as an immediate consequence of Lemma 3 , we also have $s^{\prime} \in K_{1}$, which entails that $L_{\varphi}$ is opaque w.r.t. $K_{1}$ and $\Sigma_{a}$. Hence, $K_{1}=K\left(L(G), L_{\varphi}\right)$, which according to Proposition 3 entails that $K_{1}=\mathrm{CO}-\mathrm{OP}^{\uparrow}\left(L(G), L_{\varphi}, \Sigma_{a}, \Sigma_{m}, \Sigma_{c}\right)$.

2) Assumption 2: $\Sigma_{a} \subseteq \Sigma_{c} \subseteq \Sigma_{m} \subseteq \Sigma$ : This assumption simply means that the controller can observe all the actions of the attacker and control them.

Based on proposition 4 , one can assume, without loss of generality, that $\Sigma_{m}=\Sigma$.

Proposition 6: Assume $\Sigma_{a} \subseteq \Sigma_{c} \subseteq \Sigma_{m}=\Sigma$, then $K_{1}(=\mathcal{K}(L(G)))={\mathrm{CO}-\mathrm{OP}^{\uparrow}}^{\uparrow}\left(L(G), L_{\varphi}, \Sigma_{a}, \Sigma_{m}, \Sigma_{c}\right)$ is regular and effectively computable.

Proof: We first show that $\operatorname{OP}^{\uparrow}\left(L(G), L_{\varphi}, \Sigma_{a}\right)$ is controllable with respect to $L, \Sigma_{c}$. Consider $s \in$ $\mathrm{OP}^{\uparrow}\left(L(G), L_{\varphi}, \Sigma_{a}\right)$ and $\sigma \notin \Sigma_{c}$, such that $s \sigma \in L(G)$. As $\Sigma_{a} \subseteq \Sigma_{c}, \sigma \notin \Sigma_{a}$ and then $s \sigma \in[s]_{a} \cap L(G)$ and according to Lemma 2, s $\sigma \in \operatorname{OP}^{\uparrow}\left(L(G), L_{\varphi}, \Sigma_{a}\right)$, which is then controllable w.r.t. $L, \Sigma_{c}$ and observable w.r.t. $\Sigma_{c}$ and $\Sigma_{m}$ since $\Sigma_{m}=\Sigma$. Hence,

$$
\begin{array}{r}
\mathrm{CO}^{\uparrow}\left(\mathrm{OP}^{\uparrow}\left(L(G), L_{\varphi}, \Sigma_{a}\right), L(G), \Sigma_{c}, \Sigma_{m}\right)= \\
\operatorname{OP}^{\uparrow}\left(L(G), L_{\varphi}, \Sigma_{a}\right)=K\left(L(G), L_{\varphi}\right)
\end{array}
$$

and we conclude using the result of Proposition 3.

3) Assumption 3: $\Sigma_{c} \subseteq \Sigma_{a} \subseteq \Sigma_{m} \subseteq \Sigma$ : Under this assumption, even though all actions of the attacker can be observed by the controller, only a part of them can be controlled. One can think that the controller can filter out the requests sent by the attacker to the system, whereas the outputs of the system cannot be disabled by the controller. This is for example the behavior of a firewall for Internet services.

It is easy to check that the system of Example 2, for which the fix-point computation does not terminate, fulfills the assumption of this subsection. This leads us to design a new algorithm.

Using proposition 4 , we can assume that $\Sigma_{m}=\Sigma$. We also make the following assumption without loss of generality. The system is given by a deterministic LTS $G=$ $\left(Q_{G}, \Sigma, q_{0}^{G}, \delta_{G}\right)$. The predicate $L_{\varphi}$ is specified by a complete and deterministic LTS $S_{\varphi}=\left(Q_{S}, \Sigma, q_{0}^{S}, \delta_{S}\right)$ with a set $F_{\varphi}$ of final states such that $L_{\varphi}=L_{F_{\varphi}}\left(S_{\varphi}\right)$ and $L\left(S_{\varphi}\right)=\Sigma^{*}$.

First, we compute the product of $G$ and $S_{\varphi}$ in order to tag the states in which the predicate $L_{\varphi}$ is satisfied: $G_{\varphi}=$ $G \| S_{\varphi}=\left(Q, \Sigma, q_{0}, \delta\right)$, with $Q=Q_{G} \times Q_{S}, q_{0}=\left(q_{0}^{G}, q_{0}^{S}\right)$ and $\delta$ the synchronized transition function. By denoting $F=$
$Q_{G} \times F_{\varphi}$, we get $L_{F}\left(G_{\varphi}\right)=L(G) \cap L_{\varphi}$, meaning that the execution traces that reach or go through a state of $F$ reveal $L_{\varphi}$ (note that $L\left(G_{\varphi}\right)=L(G)$, because $S_{\varphi}$ is complete). Thus, $L_{\varphi}$ is opaque w.r.t. $L\left(G_{\varphi}\right) \Leftrightarrow L_{\varphi}$ is opaque w.r.t. $L(G)$. Clearly, if $L_{\varphi}$ is non-opaque w.r.t. $L\left(G_{\varphi}\right) \backslash \Sigma^{*} \Sigma_{c} \Sigma^{*}$ and $\Sigma_{a}$, then no control can enforce the opacity of $L_{\varphi}$. So in the sequel, without loss of generality, we assume that $L_{\varphi}$ is opaque w.r.t. $L\left(G_{\varphi}\right) \backslash \Sigma^{*} \Sigma_{c} \Sigma^{*}$ and $\Sigma_{a}$. In particular, this entails that $L_{\varphi}$ is opaque w.r.t. $L\left(G_{\varphi}\right) \backslash \Sigma_{c}^{*}$.

Under this assumption, we show that the optimal opacity control may be enforced by a finite state controller, defined by a deterministic LTS $C=\left(\mathcal{Q}, \Sigma, \Theta_{0}, \delta\right)$ with the set of states

$$
\mathcal{Q}=\{(X, q): q \in X \subseteq Q\}
$$

and the initial state $\Theta_{0}=\left(X_{0}, q_{0}\right)$ specified by

$$
X_{0}=\left\{q \in Q: \exists s \in\left(\Sigma \backslash \Sigma_{a}\right)^{*}, q_{0} \stackrel{s}{\rightarrow} q\right\}
$$

Intuitively, after the execution of a trace $s$, the controller is in a state $(X, q)$ when the controlled system is in state $q$ (recall that $\Sigma_{m}=\Sigma$ ) and $X$ is the best estimate of the current state of $G_{\varphi}$ that the attacker $\mathcal{A}$ can get from the observation $P_{\Sigma_{a}}(s)$ of this execution trace. In particular, if no event in $\Sigma_{a}$ has been produced yet, the best estimate is $X_{0}$ (recall that the attacker has full knowledge of the structure of $\left.G_{\varphi}\right)^{6}$.

In the sequel, we denote $\mathcal{T}$ the set of transitions of $G_{\varphi}$.

The main task, for completing the construction of $C$, is to determine the map $\alpha: 2^{Q} \longrightarrow 2^{\mathcal{T}}$ that tells, for each state $(X, q)$ and simultaneously for all $q \in X$, which set $\alpha(X)$ of controllable transitions of $G_{\varphi}$ the controller does enable, thus

$$
\alpha(X) \subseteq \alpha_{0}(X) \triangleq\left\{q \stackrel{\sigma}{\rightarrow} q^{\prime} \in \mathcal{T}: q \in X, q^{\prime} \in Q, \sigma \in \Sigma_{c}\right\} .
$$

So, in state $(X, q)$, the controller disables the transitions $q \stackrel{\sigma}{\rightarrow}$ $q^{\prime} \in \alpha_{0}(X) \backslash \alpha(X)$, all of which are controllable.

Suppose the correct map $\alpha$ has been computed. Then the set $\mathcal{T}_{C}$ (of transitions of $C$ ) is inductively defined as the least set of transitions $(X, q) \stackrel{\sigma}{\rightarrow} C\left(X^{\prime}, q^{\prime}\right)$ such that $(X, q)$ is reachable, $q \stackrel{\sigma}{\rightarrow} q^{\prime}$, and the estimate $X^{\prime}$ of the attacker is updated from $X$ as follows:

- if $\sigma \notin \Sigma_{a}$, then $X^{\prime}=X$

- if $\sigma \in\left(\Sigma_{a} \backslash \Sigma_{c}\right)$, then

$$
X^{\prime}=\left\{\bar{q}^{\prime}: \exists \bar{q} \in X, \exists s \in \Sigma^{*}, \bar{q} \stackrel{s}{\rightarrow} \bar{q}^{\prime} \text { and } \sigma \sim_{a} s\right\}
$$

- if $\sigma \in \Sigma_{c}$, then

$$
\begin{aligned}
X^{\prime}= & \left\{\bar{q}^{\prime \prime}: \exists \bar{q} \in X: \exists \bar{q}^{\prime} \in Q, \exists s \in \Sigma^{*},\right. \\
& \left.\bar{q} \stackrel{\sigma}{\rightarrow} \bar{q}^{\prime} \in \alpha(X) \text { and } \bar{q}^{\prime} \stackrel{s}{\rightarrow} \bar{q}^{\prime \prime} \text { and } s \sim_{a} \epsilon\right\}
\end{aligned}
$$

This is coherent with the idea that the attacker has full knowledge of the structure of $C$, hence of $\alpha$.

Lemma 4: Let $(X, q) \stackrel{\sigma s}{\rightarrow} C\left(X^{\prime}, q^{\prime}\right)$ with $\sigma \in \Sigma_{a}$ and $s \in$ $\left(\Sigma \backslash \Sigma_{a}\right)^{*}$, then $\forall \overline{q^{\prime}} \in X^{\prime}, \exists \bar{q} \in X,(X, \bar{q}) \stackrel{w}{\rightarrow}_{C}\left(X^{\prime}, \bar{q}^{\prime}\right)$ with $w \sim_{a} \sigma$.

${ }^{6}$ Some states in $\mathcal{Q}$ will possibly be not reachable, but it does not matter since these states can be eliminated afterwards by trimming $C$. 
Lemma 5: Let $\Theta_{0} \stackrel{s}{\rightarrow} C(X, q)$ and $\Theta_{0} \stackrel{s^{\prime}}{\rightarrow} C\left(X^{\prime}, q^{\prime}\right)$. If $s \sim_{a} s^{\prime}$ then $X=X^{\prime}$.

Both lemmas are immediate consequences of the definition of $\mathcal{T}_{C}$.

We explain now the motivation under the definition of the map $\alpha$. Let $(X, q)$ be a reachable state of the controller, thus $q \in X$, and let $q \stackrel{\sigma}{\rightarrow} q^{\prime} \in \mathcal{T}$ with $\sigma \in \Sigma_{c}$. If, for some $s \in\left(\Sigma \backslash \Sigma_{c}\right)^{*}, q^{\prime} \stackrel{s}{\rightarrow} q " \in F$ but $\sigma s \sim_{a} s^{\prime}$ for no sequence $s^{\prime}$ such that $\bar{q} \stackrel{s^{\prime}}{\rightarrow} \bar{q}$ " $\notin F$ for some $\bar{q} \in X$, then the controller $C$ should disable $q \stackrel{\sigma}{\rightarrow} q^{\prime}$ when in state $(X, q)$. Hence, one should have $q \stackrel{\sigma}{\rightarrow} q^{\prime} \notin \alpha(X)$.

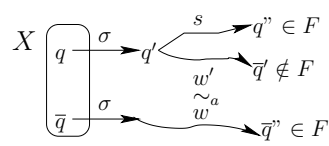

But now suppose that, for some $w, w^{\prime} \in\left(\Sigma \backslash \Sigma_{c}\right)^{*}$ and $\bar{q} \in X, \bar{q}^{\prime}, \bar{q} " \in Q, \bar{q} \stackrel{\sigma w}{\longrightarrow} \bar{q}^{\prime \prime} \in F, w \sim_{a} w^{\prime}$, and $q^{\prime} \stackrel{w^{\prime}}{\rightarrow} \bar{q}^{\prime} \notin F$ (thus $\neg\left(s \sim w^{\prime}\right)$ ). If $q \stackrel{\sigma}{\rightarrow} q^{\prime} \notin \alpha(X)$, and the attacker has full knowledge of $C$ and the map $\alpha$, the transition sequence $\bar{q} \stackrel{\sigma w}{\longrightarrow} \bar{q}$ " may now reveal the predicate $L_{\varphi}$, since the attacker knows that the masking transition sequence $q \stackrel{\sigma w^{\prime}}{\longrightarrow} \bar{q}^{\prime}$ is disabled by $C$. Therefore, $\alpha(X)$ must be computed iteratively as the limit of a decreasing chain started from the finite set $\alpha_{0}(X)$.

The definition of $\alpha(X)$ is as follows. Let $T$ range over the subsets of $\alpha_{0}(X)$, and for $\sigma \in \Sigma_{c}$, let

$$
N \operatorname{ext}(X, \sigma, T) \triangleq\left\{q^{\prime} \in Q: \exists q \in X, q \stackrel{\sigma}{\rightarrow} q^{\prime} \in T\right\}
$$

then

$$
\alpha(X) \triangleq g f p\left(\lambda T . \alpha_{0}(X) \cap \operatorname{Accept}(X, T)\right),
$$

where $\operatorname{Accept}(X, T)=T \backslash \operatorname{Bad}(X, T)$ letting

$$
\begin{aligned}
& \operatorname{Bad}(X, T) \\
& \triangleq\left\{t \in T: t=q \stackrel{\sigma}{\rightarrow} q^{\prime}, q \in X, \sigma \in \Sigma_{c},\right. \\
& P_{\Sigma_{a}}\left(L_{\left\{q^{\prime}\right\}, F}(G) \cap\left(\Sigma \backslash \Sigma_{c}\right)^{*}\right) \backslash \\
& \left.\quad P_{\Sigma_{a}}\left(L_{N \operatorname{ext}(X, \sigma, T),(Q \backslash F)}(G)\right) \neq \emptyset\right\}
\end{aligned}
$$

All transitions in $\operatorname{Bad}(X, T)$ should be disabled by control, because they may lead to a confidential information flow by triggering one controllable event followed by an uncontrollable sequence of events.

Remark 2: The controller $C$ is computed by independent iterations of the operator $\operatorname{Accept}(X, T)$ for all $X \subseteq Q$ and $T \subseteq \alpha_{0}(X)$.

We will now prove that $L_{\varphi}$ is opaque w.r.t. $L(C)$, that $L(C)$ is controllable and observable, and that it is the supremal sub-language of $L(G)=L\left(G_{\varphi}\right)$ with these properties.

Proposition 7: $L_{\varphi}$ is opaque w.r.t. $L(C)$ and $\Sigma_{a}$

Proof: Consider $s \in L(C) \cap L_{\varphi} \subseteq L(G)$.

- If $P_{\Sigma_{c}}(s)=\varepsilon$, then $s \notin \Sigma^{*} \Sigma_{c} \Sigma^{*}$. As by hypothesis $L_{\varphi}$ is opaque w.r.t. $L\left(G_{\varphi}\right) \backslash\left(\Sigma^{*} \Sigma_{c} \Sigma^{*}\right)$ and $\Sigma_{a}$, there exists $s^{\prime} \in L\left(G_{\varphi}\right)$ such that $s^{\prime} \sim_{a} s$ and $s^{\prime} \notin L_{\varphi}$. Since $\Sigma_{c} \subseteq \Sigma_{a}$, we also have $P_{\Sigma_{c}}\left(s^{\prime}\right)=\varepsilon$ and hence $s^{\prime} \in L(C)$.
- if $P_{\Sigma_{c}}(s) \neq \varepsilon$, then $s$ can be decomposed as $s=s_{1} c s_{2}$ with $c \in \Sigma_{c}$ and $s_{2} \in\left(\Sigma \backslash \Sigma_{c}\right)^{*}$. There exists $(X, q) \in \mathcal{Q}$ and $q_{1} \in Q$ such that $\Theta_{0} \stackrel{s_{1}}{\rightarrow}(X, q)$ and $\left(q \stackrel{c}{\rightarrow} q_{1}\right) \in$ $\alpha(X)$. Let $(X, q) \stackrel{c}{\rightarrow} C\left(X^{\prime}, q_{1}\right)$, thus

$$
\begin{aligned}
X^{\prime}=\left\{q^{\prime \prime} \in Q:\right. & \exists q^{\prime} \in N \operatorname{ext}(X, c, \alpha(X)), \\
& \left.\exists s \in \Sigma^{*}, q^{\prime} \stackrel{s}{\rightarrow} q^{\prime \prime} \text { and } s \sim_{a} \epsilon\right\}
\end{aligned}
$$

Assume for a contradiction that $\forall q_{1}^{\prime} \in X^{\prime}$, $\forall s_{2}^{\prime} \in\left[s_{2}\right]_{a}, \quad\left(X^{\prime}, q_{1}^{\prime}\right) \stackrel{s_{2}^{\prime}}{\rightarrow} C\left(Z, q_{2}^{\prime}\right)$ entails $q_{2}^{\prime} \in$ $F$. Then $s_{2}^{\prime} \in L_{\left\{q_{1}^{\prime}\right\}, F}\left(G_{\varphi}\right) \cap\left(\Sigma \backslash \Sigma_{c}\right)^{*}$ and $P_{\Sigma_{a}}\left(s_{2}\right) \notin P_{\Sigma_{a}}\left(L_{X^{\prime},(Q \backslash F)}\left(G_{\varphi}\right)\right)$, hence $\left(q \stackrel{c}{\rightarrow} q_{1}\right) \in$ $\operatorname{Bad}(X, \alpha(X))$, in contradiction with $(X, q) \stackrel{c}{\rightarrow} C$ $\left(X^{\prime}, q_{1}\right)$. Therefore, $\left(X^{\prime}, q_{1}^{\prime}\right) \stackrel{s_{2}^{\prime}}{\rightarrow} C\left(Z, q_{2}^{\prime}\right)$ for some $q_{1}^{\prime} \in$ $X^{\prime}, s_{2}^{\prime} \in\left[s_{2}\right]_{a}$, and $q_{2}^{\prime} \notin F$. Now, $(X, q) \stackrel{c}{\rightarrow} C\left(X^{\prime}, q_{1}\right)$ and $q_{1}^{\prime} \in X^{\prime}$ entail that $(X, \bar{q}) \stackrel{c s^{\prime}}{\rightarrow} C\left(X^{\prime}, q_{1}^{\prime}\right)$ for some $\bar{q} \in X$ and $s^{\prime} \in\left(\Sigma \backslash \Sigma_{a}\right)^{*}$ (Lemma 4$)$, and $\bar{q} \in X$ entails that $\Theta_{0} \stackrel{s_{1}^{\prime}}{\rightarrow} C(X, \bar{q})$ for some $s_{1}^{\prime} \sim_{a} s_{1}$ (Lemma 4). Altogether, $s=s_{1} c s_{2} \sim_{a} s_{1}^{\prime} c s^{\prime} s_{2}^{\prime} \in L(C) \backslash L_{\varphi}$. So $L_{\varphi}$ is opaque w.r.t. $L(C)$ and $\Sigma_{a}$.

Proposition 8: $L(C)$ is controllable w.r.t. $L\left(G_{\varphi}\right)$ and $\Sigma_{c}$. Proof: Let $s \in L(C)$ and $\sigma \in \Sigma \backslash \Sigma_{c}$ such that $s \sigma \in$ $L\left(G_{\varphi}\right)$. Then, $\exists(X, q) \in \mathcal{Q}$ such that $\Theta_{0} \stackrel{s}{\rightarrow}(X, q)$ in $C$ and $\exists q^{\prime} \in Q$ such that $q \stackrel{\sigma}{\rightarrow} q^{\prime}$ in $G_{\varphi}$. Since $\sigma \notin \Sigma_{c},(X, q) \stackrel{c}{\rightarrow} C$ $\left(X^{\prime} q^{\prime}\right)$ for some $q^{\prime}$ by definition of $\mathcal{T}_{C}$, hence $s \sigma \in L(C)$.

Theorem 1: Assume that $\Sigma_{c} \subseteq \Sigma_{a} \subseteq \Sigma_{m}=\Sigma$, then

$$
L(C)=\mathrm{CO}-O P^{\uparrow}\left(L(G), L_{\varphi}, \Sigma_{a}, \Sigma, \Sigma_{c}\right)
$$

Proof: Let $K=\mathrm{CO}-\mathrm{OP}^{\uparrow}\left(L(G), L_{\varphi}, \Sigma_{a}, \Sigma, \Sigma_{c}\right)$. Since $L_{\varphi}$ is opaque w.r.t. $L(C)$ and $\Sigma_{a}, L(C)$ is controllable w.r.t. $L\left(G_{\varphi}\right)$ and $\Sigma_{c}$, and $L(C)$ is observable w.r.t. $\Sigma_{m}$ and $L(G)$ (as $\Sigma_{m}=\Sigma$ ), we have $L(C) \subseteq K$.

It remains to prove that $K \subseteq L(C)$. We proceed by contradiction. Let $s \in K \backslash L(C)$. This sequence can be decomposed as $s=s_{1} \sigma s_{2}$, where $s_{1}$ is the longest prefix of $s$ such that $\left[s_{1}\right]_{a} \cap K=\left[s_{1}\right]_{a} \cap L(C)$, hence $s_{1} \in L(C)$ (since $s_{1} \in K$ ), $\left[s_{1} \sigma\right]_{a} \cap K \neq\left[s_{1} \sigma\right]_{a} \cap L(C)$ (by definition of $s_{1}$ ), and $\sigma \in \Sigma_{a}$ (because $\left[s_{1} \sigma\right]_{a} \neq\left[s_{1}\right]_{a}$ ).

Since $L(C) \subseteq K,\left[s_{1} \sigma\right]_{a} \cap L(C) \subset\left[s_{1} \sigma\right]_{a} \cap K$ and one can find $u=u_{1} \sigma u_{2}$ in $\left[s_{1} \sigma\right]_{a} \cap K$, with $u_{1} \sim_{a} s_{1}$ and $u_{2} \sim_{a} \epsilon$, such that $u_{1} \sigma u_{2} \notin L(C)$. As $s_{1} \in L(C) \cap K, u_{1} \sim_{a} s_{1}$ and $u_{1} \in K$, necessarily $u_{1} \in L(C)$ by definition of $s_{1}$. As $u_{1} \in L(C), u_{2} \in\left(\Sigma \backslash \Sigma_{c}\right)^{*}, u_{1} \sigma u_{2} \in L\left(G_{\varphi}\right) \backslash L(C)$, and $L(C)$ is controllable, necessarily $u_{1} \sigma \notin L(C)$ and $\sigma \in \Sigma_{c}$.

Since $u_{1} \in L(C)$, there exists $(X, q) \in \mathcal{Q}$ such that $\Theta_{0} \stackrel{u_{1}}{\rightarrow}$ $(X, q)$. By construction of $C, q_{0} \stackrel{u_{1}}{\rightarrow} q$ in $G_{\varphi}, q \in X$, and $X=\left\{q_{1} \in Q: \exists u \in\left[u_{1}\right]_{a} \cap L(C), q_{0} \stackrel{u}{\rightarrow} q_{1}\right\}$. It also follows from the construction of $C$ that

$$
\begin{aligned}
\alpha(X)= & \left\{q_{1} \stackrel{c}{\rightarrow} q_{2}: q_{1} \in X, q_{2} \in Q, c \in \Sigma_{c}\right. \text { s.t. } \\
& \left.\exists u \in\left[u_{1}\right]_{a} \cap L(C), q_{0} \stackrel{u}{\rightarrow} q_{1} \text { and } u c \in L(C)\right\}
\end{aligned}
$$

and moreover, for all $q_{1} \stackrel{c}{\rightarrow} q_{2} \in \alpha(X), \forall u \in\left[u_{1}\right]_{a} \cap L(C)$, $q_{0} \stackrel{u}{\rightarrow} q_{1} \Rightarrow u c \in L(C)$. Therefore, $u_{1} \in\left[u_{1}\right]_{a} \cap L(C), q_{0} \stackrel{u_{1}}{\rightarrow}$ 
$q, \sigma \in \Sigma_{c}$, and $u_{1} \sigma \notin L(C)$ entail that $\alpha(X)$ contains no transition $q \stackrel{\sigma}{\rightarrow} q^{\prime}$.

Consider now the alternative set of transitions

$$
\begin{aligned}
\beta=\left\{q_{1} \stackrel{c}{\rightarrow} q_{2} \quad: q_{1} \in X, q_{2} \in Q, \sigma \in \Sigma_{c}\right. \\
\text { s.t. } \left.\exists u \in\left[u_{1}\right]_{a} \cap K, q_{0} \stackrel{u}{\rightarrow} q_{1} \text { and } u \sigma \in K\right\}
\end{aligned}
$$

Then clearly $\alpha(X) \subseteq \beta$ (because $L(C) \subseteq K$ ) and $\beta$ contains a transition $q \stackrel{\sigma}{\rightarrow} q^{\prime}$ (because $q_{0} \stackrel{u_{1}}{\rightarrow} q, u_{1} \sigma \in K$, and $K \subseteq$ $\left.L\left(G_{\varphi}\right)\right)$. Therefore, $\alpha(X) \subset \beta$.

In order to complete the proof, we will show that $\left(q_{1} \stackrel{c}{\rightarrow}\right.$ $\left.q_{2}\right) \notin \operatorname{Bad}(X, \beta)$ for all $c \in \Sigma_{c}$ and $\left(q_{1} \stackrel{c}{\rightarrow} q_{2}\right) \in \beta$, entailing that $\operatorname{Accept}(X, \beta)=\beta \backslash \operatorname{Bad}(X, \beta)=\beta$, and hence that $\beta \subseteq \alpha(X)$ in view of the greatest fixpoint definition of $\alpha(X)$, resulting in a contradiction with $\alpha(X) \subset \beta$.

Let $\left(q_{1} \stackrel{c}{\rightarrow} q_{2}\right) \in \operatorname{Bad}(X, \beta)$. Recalling that $\operatorname{Bad}(X, \beta) \subseteq$ $\beta$ and that $\left[u_{1}\right]_{a}=\left[s_{1}\right]_{a}$, let $u \in\left[u_{1}\right]_{a} \cap L(C)=\left[u_{1}\right]_{a} \cap K$ such that $\Theta_{0} \stackrel{u}{\rightarrow} C\left(X, q_{1}\right)$ and $u c \in K$. As $\left(q_{1} \stackrel{c}{\rightarrow} q_{2}\right) \in$ $\operatorname{Bad}(X, \beta)$, there must exist $v \in\left(\Sigma \backslash \Sigma_{c}\right)^{*}$ such that $u c v \in$ $L_{\varphi} \cap L\left(G_{\varphi}\right) . K$ is controllable, hence $u c v \in K . L_{\varphi}$ is opaque w.r.t. $K$ and $\Sigma_{a}$, then $\exists w \in[u c v]_{a} \cap K$ such that $w \notin$ $L_{\varphi}$. As $w \in[u c v]_{a}$ and $c \in \Sigma_{c} \subseteq \Sigma_{a}$, there should exist $w_{1}, w_{2} \in \Sigma^{*}$ such that $w=w_{1} c w_{2}$ with $w_{1} \sim_{a} u \sim_{a} u_{1}$ and $w_{2} \sim_{a} v$. Now, $w_{1} \in\left[u_{1}\right]_{a} \cap K \Rightarrow w_{1} \in\left[u_{1}\right]_{a} \cap L(C) \Rightarrow$ $\exists q_{3} \in X, \Theta_{0} \stackrel{w_{1}}{\rightarrow}\left(X, q_{3}\right)$ in $C$, by Lemma 5. As $w_{1} c \in K$, there must exist $q_{4} \in Q$ such that $\left(q_{3} \stackrel{c}{\rightarrow} q_{4}\right) \in \beta$ and thus $q_{4} \in N \operatorname{ext}(X, c, \beta)$. Now, $v \in L_{\left\{q_{2}\right\}, F}(G) \cap\left(\Sigma \backslash \Sigma_{c}\right)^{*}$, $w_{2} \in L_{\left\{q_{4}\right\},(Q \backslash F)}(G) \cap\left(\Sigma \backslash \Sigma_{c}\right)^{*}$, and $v \sim_{a} w_{2}$, so based on the definition of the $\operatorname{Bad}$ operator, $\left(q_{1} \stackrel{c}{\rightarrow} q_{2}\right) \notin \operatorname{Bad}(X, \beta)$, which is the expected contradiction.

Example 3: To illustrate the algorithm, let us come back to our previous example, where $F=\{3,11\}$.

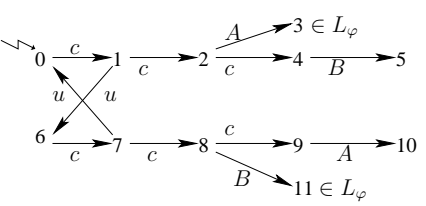

Fig. 6. $L(G)$ and $L_{\varphi}$

At the first step of the computation of $L(C)$, we get $X_{0}=$ $\left.\{0\}, \Theta_{0}=\left(X_{0}, 0\right)\right)$ and $\alpha_{0}\left(X_{0}\right)=\{(0 \stackrel{c}{\rightarrow} 1)\}$. Now, we also have $P_{\Sigma_{a}}\left(L\left(G_{\varphi}, 1, F\right) \cap\left(\Sigma \backslash \Sigma_{c}\right)^{*}\right)=\emptyset$, implying $(0 \stackrel{c}{\rightarrow} 1) \notin$ $\operatorname{Bad}\left(X_{0}, \alpha_{0}\left(X_{0}\right)\right)$, and thus $\alpha\left(X_{0}\right)=\{(0 \stackrel{c}{\rightarrow} 1)\}$. Thus, in $C$, we have $\Theta_{0} \stackrel{c}{\rightarrow} C(\{1,6\}, 1)$.

Further, for $X_{1}=\{1,6\}$, we get $\alpha_{0}\left(X_{1}\right)=\{(1 \stackrel{c}{\rightarrow} 2),(6 \stackrel{c}{\rightarrow}$ $7)\}, N \operatorname{ext}\left(X_{1}, c, \alpha_{0}\left(X_{1}\right)\right)=\{2,7\}$,

$$
P_{\Sigma_{a}}\left(L\left(G_{\varphi}, 2, F\right) \cap\left(\Sigma \backslash \Sigma_{c}\right)^{*}\right)=\{A\}
$$

$$
\begin{aligned}
& \text { and } P_{\Sigma_{a}}\left(L\left(G_{\varphi}, N \operatorname{ext}\left(X_{1}, c, \alpha_{0}\left(X_{1}\right)\right), Q \backslash F\right)\right. \\
& =(c c)^{+} .(A+c B)+\overline{c B}+c c
\end{aligned}
$$

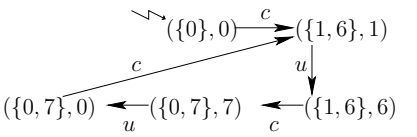

Fig. 7. The corresponding supervisor $C$
Thus, $1 \stackrel{c}{\rightarrow} 2 \in \operatorname{Bad}\left(X_{1}, \alpha_{0}\left(X_{1}\right)\right)$ and $(1 \stackrel{c}{\rightarrow} 2) \notin$ $\alpha_{1}\left(X_{1}\right)$. Finally, it can be shown that $\{(6 \stackrel{c}{\rightarrow} 7)\}=\alpha\left(X_{1}\right)$. The other values of $\alpha(X)$ for $X \in \mathcal{Q}$ can be computed similarly. The resulting $C$ is given by the LTS of Figure $7 \diamond$ Acknowledgment: The authors would like to thank the reviewers and Thierry Jéron for their helpful comments.

\section{CONCLUSiON}

Given a finite transition system $G$ over $\Sigma$ and a regular predicate $L_{\varphi} \subseteq \Sigma^{*}$, we have addressed the problem of computing a supervisor $C$ that enforces the opacity of $L_{\varphi}$ against an attacker with alphabet $\Sigma_{a} \subseteq \Sigma$, supposedly trying to push $G \times C$ to reveal $L_{\varphi}$ (i.e. to produce an execution $s$ such that $P_{\Sigma_{a}}(s)=P_{\Sigma_{a}}\left(s^{\prime}\right) \Rightarrow s^{\prime} \in L_{\varphi}$ for all $s^{\prime} \in$ $L(G \times C))$. We have shown how computing the optimal finite state supervisor $C$ with controllable (observable) alphabet $\Sigma_{c}$ $\left(\Sigma_{m}\right)$ in all cases where $\Sigma_{c} \subseteq \Sigma_{m}$ and $\Sigma_{a}$ compares with both.

We do not know yet whether the technical answer we have provided to this problem can be extended to cope with more complex situations, such as for instance the case where $\Sigma_{c} \subseteq \Sigma_{m}$ and $\Sigma_{a} \subseteq \Sigma_{m}$ (the algorithm defined in $\mathrm{V}$ B.3 may not give the optimal supervisor in this case), or the case where one wants to enforce simultaneously the opacity of two predicates with respect to two attackers with different interfaces. An important question to be studied before applications are considered is the relation between opacity and finite state abstraction of possibly infinite state systems. Another topic of interest is the preservation of opacity by algebraic operations of system composition.

\section{REFERENCES}

[1] L. Mazaré, "Using unification for opacity properties," in Proceedings of the 4th IFIP WG1.7 Workshop on Issues in the Theory of Security (WITS'04), Barcelona (Spain), 2004, pp. 165-176.

[2] J. Bryans, M. Koutny, L. Mazaré, and P. Y. A. Ryan, "Opacity generalised to transition systems," in Revised Selected Papers of the 3rd International Workshop on Formal Aspects in Security and Trust (FAST'05), ser. Lecture Notes in Computer Science, T. Dimitrakos, F. Martinelli, P. Y. A. Ryan, and S. A. Schneider, Eds., vol. 3866. Newcastle upon Tyne, UK: Springer, 2006, pp. 81-95.

[3] R. Alur, P. Černý, and S. Zdancewic, "Preserving secrecy under refinement," in ICALP '06: Proceedings (Part II) of the 33rd International Colloquium on Automata, Languages and Programming. Springer, 2006, pp. 107-118.

[4] J. Dubreil, T. Jéron, and H. Marchand, "Construction de moniteurs pour la surveillance de propriétés de sécurité," in 6ème Colloque Francophone sur la Modélisation des Systèmes Réactifs, Lyon, France, October 2007.

[5] E. Badouel, M. Bednarczyk, A. Borzyszkowski, B. Caillaud, and P. Darondeau, "Concurrent secrets," Discrete Event Dynamic Systems, vol. 17, pp. 425-446, 2007, extended version of a Wodes'06 paper.

[6] F. B. Schneider, "Enforceable security policies," ACM Trans. Inf. Syst. Secur, vol. 3, no. 1, pp. 30-50, 2000.

[7] J. Ligatti, L. Bauer, and D. Walker, "Edit automata: enforcement mechanisms for run-time security policies," Int. J. Inf. Sec., vol. 4, no. $1-2$, pp. 2-16, 2005.

[8] C. Cassandras and S. Lafortune, Introduction to Discrete Event Systems. Kluwer Academic Publishers, 1999.

[9] R. D. Brandt, V. Garg, R. Kumar, F. Lin, S. I. Marcus, and W. M. Wonham, "Formulas for calculating supremal controllable and normal sublanguages," Systems \& Control Letters, vol. 15, no. 2, pp. 111-117, 1990.

[10] A. Tarski, "A lattice-theoretical fixpoint theorem and its applications," Pacific Journal of Mathematics, vol. 5, pp. 285-309, 1955. 